Mr. Forbes exactly gives the difference, as thinner, more wiry, and high pitched; it is also shorter, at least in the wild $G$. ferrugina. These I have often heard crowing, and shot in the extreme east of Asam, where for a very large area, on the Upper Diking River, and across Patkai, there are no inhabitants.

This same G. ferrugina is, however, to be found wild all over Asam, and the countries around; eggs found in the jungles are often hatched under domestic fowls, and hence these are frequently crossed, and the crow of the cock varies much in consequence.

But the difference between the wild $G$. ferrugina and our "barn-door" cock, in this particular, is so well marked that it could invariably be detected.

I may perhaps mention a curious sight I saw last year, within Ioo yards of my bungalow, in the evening. A cloud of white ants were rising on the air, in the main road, and a jackal and jungle cock were busy eating the "neuters" swarming all over the ground; presently another jackal joined and the cock was between them : all were so busy feeding that they took no notice of each other, the jackals often lying on their bellies, while the cock moved about between them, at 2 or 3 yards only. By this time 15 or 20 people were looking on and laughing. Suddenly a third, younger jackal, joined the group, and after eating the ants a short time, and walking about like the others, dropped into the ditch and stalked the cock, crouching close to him. The latter at once flew, and made a bee line for the forest 400 yards off. The total area of the ants was about 20 feet by 8 only.

Sibsagar, Asam, March 27.

S. E. PEAL.

\section{Antipathy [?] of Birds for Colour.}

WiTH regard to the destruction of the yellow crocus by the sparrow, mentioned by your correspondent "M. H. M." in NATURE, vol. xliii. p. 558, this bird appears to have a predilection for yellow. In an article on '“Birds' Nests and Nestbuilding," in the Animal World, present number, an instance is given of sparrows using the flowers of the laburnum for their nest. Only lately I have been watching them picking out the yellow centres of the daisy, but in this case it was for food, and I am inclined to believe that some portion of the crocus is also eaten. At this time of the year they are well known to be partial to buds and flowers of different kinds-for instance, the blossoms of the gooseberry bushes.

Doubtiess, the bright yellow colour attracts the attention of this now much censured bird, so omnivorous in his tastes and such a general scavenger, and therefore not wholly to be condemned.

Clevedon, April 28. T. B. J.

\section{The Destruction of Fish by Frost.}

Referring to Prof. Bonney's letter in Nature, vol. xliii. p. 295, regarding the destruction of fish by frost, and in which he asks for information from more northern latitudes, I may say that during the winter of $1885-86$, at Cape Prince of Wales, Hudson's Strait, when the thickness of ice in a small lake was being measured, live fish were often seen; and upon the last occasion, when the ice measured six feet and half an inch, several were thrown up with the water that, upon our cutting through, immediately overflowed. These fish were about an inch and a half in length and were extremely lively. I may add that during the summer both feeder and outlet of the lake averaged about eight inches in depth and the lake nine feet in its deepest part. The former ceased to flow on November 8, when, too, ice, fourteen inches in thickness, covered the lake.

Meteorological Service of Canada, Toronto, April 16.

\section{The Flying to Pieces of a Whirling Ring.}

WITH reference to the recent discussion in your columns on the whirling of steel bands, the fullowing results will be of interest.

A weldless steel flask, with spherical body 12 inches in diameter and $\frac{8}{8}$ inch thick, constructed for use in a centrifugal milk separator, to revolve about its axis of symmetry at a normal speed of 7000 revolutions per minute, was whirled at a gradually increasing speed, with a view to ascertaining the "bursting" velocity.

At 16,000 revolutions per minute the body of the flask had NO. I I 24 , VOL. 44] bulged 2 inches in diameter : this is equivalent to an extension of 17 per cent. of the circumference ; the peripheral speed being 840 feet per second, and the tension $3 r^{\prime} 5$ tons per square inch.

The experiment was not continued, as it was considered suffi. ciently satisfactory, and the bulged flask is kept as a curiosity.

McGill University, Montreal. Chas. A. Carus-Wilson.

\section{HERTZ'S EXPERIMENTS. \\ III.}

$\mathrm{N}$ the last article the principles upon which a rapidly vibrating electric oscillator should be constructed were considered, and how the sudden break-down of the air gap enabled these rapid vibrations to be started. It is probable that this break-down occurs in a time smaller than the thousand millionth of a second. How very rapid interatomic motions must be!

Consider now the principles on which an apparatus is to be constructed to receive the vibrations produced by this oscillator. We may observe in the first place that as we are dealing with a succession of impulses at equal intervals of time we can utilize resonance to accumulate the effect of a single impulse. Resonance is used in an immense variety of circumstances to accumulate the effect of a series of impulses, and is avoided in another immense variety of circumstances to prevent accumulating the effect of a series of impulses. We see, we hear, we photograph by using it; we use it to make musical sounds, to keep clocks and watches going, to work telegraphs. By avoiding it carriages drive safely over rough roads, ships navigate the seas, the tides do not now overwhelm the land, the earth and planets preserve their courses round the sun, and the solar system is saved from destruction. Resonance may be thus described :If a system is able to vibrate by itself in any way, and if we give it a series of impulses, each tending to increase the vibration, the effect will be cumulative, and the vibration will increase. To do this the impulses must be well timed, at intervals the same as the period of vibration of the system itself. Otherwise some of the impulses will tend to stop the vibration, and only some to increase it, and on the whole the effect will be small. In order to use resonance in the construction of the detector of waves of electric force, we must make our detector so as to be capable of an electric vibration of the same period as the generator of the waves. If we do this we may expect the currents produced in it to be increased by each wave, and thus the electrification at its ends to increase, and so increase the chance of our being able to produce a visible spark. Two ways of using a detector have been mentioned. One is to observe the heating of a conductor by the current in it, and the other to observe a spark due to the electrification at the end of the conductor. The latter is the most sensitive and has been most frequently employed, and is the method first employed by Hertz. Two forms of detector may be used for observing sparks. One form consists of a single conductor bent into a circle with its two extremities very close together. An electric charge can oscillate from one end of this to the other round the circle and back again. If the circle be the proper size, about $70 \mathrm{~cm}$. in diameter for the large sized oscillator and about $8 \mathrm{~cm}$. in diameter for the smaller sized one described in the last article, the period of oscillation of this charge will be the same as that of the charge on the generator of the waves, and its oscillation will be increased by resonance until, if the ends of the circular wire be close enough together, the opposite electrification of the ends will become great enough to cause a spark across the gap. The other form of detector depends on using two conductors, each of which has the same period of electric oscillation as the oscillations we wish to detect. These

\footnotetext{
¿ Continued from p. I4.
} 
are placed in such a position that an end of one is near that end of the other which will at any time be oppositely electrified. For example, if the electric force in our waves be in vertical lines, then if we place two elongated conductors, one vertically above the other and separated by a very small air space, the electric force alternating up and down will cause currents to run up and down the conductors simultaneously, and the upper ends of both will be similarly electrified at any instant, while the lower end of the upper one will always be oppositely electrified to the upper end of the lower conductor, and if these two points, or two short wires connected with them, be close enough together, a spark will pass from one to the other whenever the electric force sets up these electric oscillations in the conductor. Thus this apparatus is a detector of the electric force. Whenever there is a spark we may be sure that there is electric force, and whenever we cannot get a spark we may be sure that there is either no electric force or anyway too little to produce sparks. The apparatus will be more sensitive for electric forces that oscillate at the same rate as the natural vibration of the electric charge on the conductor, because the effect of each impulse will then add to that of the last ; resonance will help to make the electrifications great, and so there will be a better chance of our being able to produce a spark. We may weaken the strength of this air gap by reducing the pressure of the air in it. To do this the ends of the conductors, or wires connected with them, must lead into an exhausted air vessel, such as a Geissler's tube. There is no doubt that much longer sparks may thus be produced, but they are so dim and diffused that when dealing with very minute quantities of electricity those sparks in a vacuum are not more easily seen than the smaller and intenser sparks in air at atmospheric pressure. The additional complication and difficulty of manipulation from having the terminalsin a vacuum are not compensated for by any advantages. This whole detecting apparatus works on somewhat the same principle as a resonator of definite size connected with one's ear when used to detect a feeble note of the same pitch as the resonator. Such a resonator might very well be used to find out where this note existed and where it did not. It would detect where there were compressions and rarefactions of the air producing currents of air into and out of your ear. In the same way the conductor sparking tells where there are alternating electric forces making currents alternately up and down the conductor, and ultimately electrifying the end enough to make it spark. In the sound resonator there is nothing exactly like this last phenomenon. We have much more delicate ways of detecting the currents of air than by making them break anything. If anybody would allow the electric currents from a Hertzian detector to be led directly into the retina of his eye, it would probably be a very delicate way of observing, though even in this direct application of the current to an organ of sense it is possible that these very rapidly alternating currents might fail to produce any sensible effect, for they are not rapid enough to produce the photochemical effects by which we see.

To recapitulate the arrangements proposed in order to detect whether electric force is propagated with a finite velocity, and if possible to measure it if finite. It is proposed to create electric oscillations of very great rapidity, oscillating some four or five hundred million times per second, and it is expected thereby to produce waves of electric force whose length will be less than a metre if they are propagated with the velocity of light. It is proposed to do this by causing an electric charge to oscillate backwards and forwards between two conductors, and across an air gap between them. This oscillating charge is to be started by charging the conductors, one positively and the other negatively, until they discharge by a spark across this air gap. By making the conductors small, and the distance the charge has to go from one to the other small, the rate of oscillation of the charge can be made as great as we require. If waves are produced by this arrangement, we can reflect them at the surface of a large conducting sheet, and then loops and nodes will be produced where the incident and reflected waves co-exist. The Ioops will be places where the alternating electric forces are great, while at the nodes there will be no electric forces at all. In order to detect where there are these alternating electric forces and where there are none, it is proposed to use either a single wire bent nearly into a circle, with a very minute air gap between its ends, or else two conductors placed end to end, with a minute air gap between their ends. In either case, if the natural period of vibration of a charge on the single conductor, or on each of the conductors in the second arrangement, is the same as the rate of alternation of the electric force we wish to detect, there may be sufficient electrification of the neighbouring ends to cause a spark across the minute air gap. We are thus in possession of a complete apparatus for determining whether electric waves are produced, and what their wave-length is.

The experiment is conducted as follows:-

The two conductors which are to generate the waves are placed, say, one above the other, so that the electric charge will run up and down in a vertical line across the spark gap between them. They might be placed horizontally or in any other line, but for definiteness of description it is well to suppose some definite position. We may call them $A$ and $B$. They are terminated in polished knobs, between which the spark passes. A and $\mathrm{B}$ are connected with the terminals of a Ruhmkorff coil, or a Wimshurst or other apparatus by which a succession of sparks may be conveniently made to pass from $A$ to B. Before the spark passes, $\mathrm{A}$ and $\mathrm{B}$ are being electrified, and when the spark occurs the electricity on $A$ rushes over to $\mathrm{B}$, and part of it charges $\mathrm{B}$, while the electricity on $\mathrm{B}$ rushes across the spark, and partly charges A, this taking place alternately up and down. Each time there is less electricity, for some is neutralized during each oscillation by the opposite charge; for energy is being spent, some in overcoming the resistance of the spark gap, i.e. in producing the heat developed there, and some in producing electric waves in the surrounding medium. Thus the electric energy of the two oppositely charged bodies A and B is gradually dissipated, and one way of describing this is to say that the two opposite electric charges combine and neutralize one another. This whole language of talking of electric charges on bodies, and electric currents from one to the other, of electric charges neutralizing one another, and so forth, is not in accordance with the most recent developments of electro-magnetic theory. At the same time, those for whom these articles are written are familiar with this language, and with the view of the subject that it is framed to suit, while they are unfamiliar with ether electrically and magnetically strained and thereby the seat of electric and magnetic energy, and consequently it would have added very much to their difficulty in grasping the details of a complicated question if it had been described in unfamiliar terms, and from an unfamiliar point of view.

The electric force in the neighbourhood of the vertical generator will lie in vertical planes through it, and as $A$ and $B$ are alternately positive and negative, the electric force will alternately be from above downwards, and from below upwards. If, then, this force is propagated outwards in a series of waves, we may expect that all round our generator waves of electric force will be diverging; waves in which the force will be alternately down and up. The state of affairs might be roughly illustrated by elastic strings stretched out in every direction from our generator. If their ends at the generator be moved alternately down and up, waves will be propa-

NO. I I 24, VOL. 44] 
gated along the strings, waves of alternate motion down and up.

In order to reflect these waves, we require a metallic sheet of considerable area some two or three wave-lengths away from the generator; so far away in order that we may have room for our detector to find the loops and nodes formed every half wave-length where the outgoing waves meet those reflected from the screen. Not too far away, or our waves will be too feeble even at the loops to affect our detector. The waves are thrown off all round, but are most intense in the horizontal plane through the spark, so that our detector had better be placed as near to this plane as possible. The detector may be either a very nearly closed circle of wire, or two conductors, each somewhat longer and thinner than the combined lengths of the generating conductors, and placed vertically over one another, and separated by a minute air gap. As the theory of this latter form of detector is simpler than that of the circle, it will simplify matters to consider it alone. The two conductors should each have a period of electrical oscillation up and down it, the same as that of the charges on the generator. The generator consists of two conductors certainly, but then during the time the spark lasts they are virtually one conductor, being connected by the spark across which the electric charges are rushing alternately up and down. Hence the period of oscillation of the charges on the generator corresponds to that on a single conductor of the same size as its two parts combined. Various experiments have been made as to the best form for these conductors that form the detector. They might be made identical with the generator, only that the spark gap in the generator should be represented by a connecting wire. They may be longer and thinner. If longer, they should be thinner, or they will not have the same period of vibration. On the whole, the best results have been got with conductors somewhat longer and thinner than the generator. It is not generally convenient that the spark between the two conductors that form the detector should take place directly from one to the other. It is not easy to make arrangements by which distance apart of these conductors can be regulated sufficiently accurately. The most convenient way is to connect the lower end of the upper conductor and the upper end of the lower one each with a short thin wire leading, one to a fixed small knob, and the other to a very fine screw impinging on the knob. The screw may then be used to adjust the spark gap between it and the small knob with great accuracy. This spark gap must be very small indeed, if delicate work be desired. A thousandth of a centimetre would be a fair-sized spark gap. The minute sparks that are formed in these gaps when doing delicate work are too faint to be seen, except in a darkened room. Having placed the detector in position between the generator and the screen, the difficult part of the observation begins. It is heartrending work at first. A bright spark now and then arouses hope, and long periods of darkness crush it again. The knobs of the generator require repolishing; the spark gap of the detector gets closed up ; dust destroys all working; and not without much patience can the art be attained of making sure of getting sparks whenever the conditions are favourable, though it is easy enough not to get sparks when the conditions are unfavourable. Before making any measurements, all this practice must be gone through. It is hard enough with the success of others before us to encourage us, with their advice to lead us, with a clear knowledge of what is to be expected to guide us. How much credit, then, is due to Hertz, who groped his way to these wonderful experiments from step to step, without the success of others to encourage him, without the advice of others to lead him, without any certainty as to what was to be expected to guide him. Patiently, carefully, through many by-paths, with constant watchfulness, and checking every advance by repeated and varied experiments, Hertz worked up to the grand simplicity of the fundamental experiment in electricity that is engaging our attention.

Having gained command over the apparatus, we may look about for places where sparks occur easily, and for others where they cannot be produced. Two or three places may be found where no sparks can be observed. These places will be found to be nearly equidistant. They are the nodes we are in search of. The distance between any pair is half the distance an electric wave is propagated during the period of an oscillation. Their presence proves that the electric force is not propagated instantaneously, but takes time to get from place to place. If the electric force were propagated instantaneously, there might be one place where the action of the currents induced in our reflecting sheet neutralized the direct action of our generator; but there could not be a series of two or more such places between the generator and the reflecting sheet. That there are more than one proves that electric force is propagated from place to place, and does not occur simultaneously everywhere. It sets the crowning stone on Maxwell's theory that electric force is due to a medium. Without a medium there can be no propagation from place to place in time. It only remains to confirm by calculation that the rate of propagation is the same as that of light. This is a complicated matter. It involves the question of how fast should, on any theory, the charge oscillate up and down a conductor The problem has only been accurately solved in a few special cases, such as that of a sphere by itself. The conductors that have been employed are not this shape, are not by themselves, and so only rough approximations are possible as to the rate at which these oscillations occur. Knowing the wave-length will not determine the velocity of propagation unless we know the period of vibration; and consequently this direct measure of the velocity has only been roughly made; but it agrees as accurately as could be expected with Maxwell's theory that it must be the same as the velocity of light if electrical phenomena are due to the same medium as light. The conviction that more accurate determinations will confirm this agreement is founded upon safe ground. It was pointed out that the ether that transmits light and is set in vibration by the molecules of matter can hardly avoid moving them itself. This ether can hardly help having other properties than merely transmitting a comparatively small range of vibrations. It can hardly help producing other phenomena. When it has been shown that, if there is a medium concerned in conveying electric and magnetic actions, it must possess properties which would enable it to transmit waves like light ; and when it has been shown that there is a medium concerned in conveying electric and magnetic actions, and that the rate at which they are conveyed is approximately the same as the rate at which light is propagated; the conclusion is almost unavoidable that we are dealing with the same medium in both cases, and that future experiments, capable of accurate calculation and observation, will confirm the conclusion that electric force is propagated through, and by means of, the luminiferous ether with the velocity of light. We really know very little about the nature of a wave of light. We know a great deal more about electric and magnetic forces, and much may be learnt as to the nature of a wave of light by studying it under the form of a wave of electric force. The waves produced by the Hertzian generator may be a metre long or more. The difficulty is to get them short enough. We know a good deal about how they are produced, and from this, and also by means of suitable detectors, we can study a great deal about their structure. They are truly very long waves of light. Atoms are Hertzian generators whose period of vibration is hundreds of millions of millions per second. A Hertzian generator may vibrate rapidly, but it is miserably slow compared 
with atoms. And yet the wonder is that atoms vibrate so slowly. If a Hertzian generator were, say, $10^{-7} \mathrm{~cm}$. long, about the size of a good big atom, its period of vibration would be some hundreds of times too rapid to produce ordinary light. Atoms are probably complicated Hertzian generators. By making a complicated shape, as, for example, a Leyden jar, a small object may have a slow period of vibration. All that is required is that the capacity and self-induction may be large in comparison with the size of the conductor. We saw that these rapidly vibrating generators have but little energy in them : they rapidly give out their energy to the ether near them. This is also the case with atoms. These, when free to radiate, give up their energy with wonderful rapidity. How short a time a flash of lightning lasts! It is hardly there but it is gone : the heated air molecules have so suddenly radiated off their energy. The reason why atoms in the air, for instance, do not radiate away their energy like this is because all their neighbours are sending them waves. Each molecule is a generator, but it is a detector as well. It is kept vibrating by its neighbours : it occupies a part of the ether that is in continual vibration, and so the atom itself vibrates. As each atom can radiate so rapidly, it must be a good detector: its own vibrations must be very much controlled by the neighbourhood it finds itself in ; and as the waves of light are very long compared with the distances apart of molecules, those in any neighbourhood are probably, independently of their motions to and fro, each vibrating in the same way. It is interesting to calculate how much of the energy in the air is in the form of vibrations of the ether between the molecules of air. A rough calculation shows that in air at the ordinary density and temperature only a minute fraction of the total energy in a cubic centimetre is in the ether; but when we deal with high temperatures, such as exist in lightning-flashes and near the sun, and with very small densities, there may be more energy in the ether than in the matter within each cubic centimetre. All this shows how wide-reaching are the results of Hertz's experiments. They teach us the nature of waves of light. We can learn much by considering how the waves are generated. Let us consider what goes on near the generator, consisting of two conductors, $A$ and $B$, sparking into one another. Before each spark, and while A and B are being comparatively slowly what is called charged with electricity, the ether around and between them is being strained. The lines of strain are the familiar tubes of electric force. If $\mathrm{A}$ be positive, these tubes diverge from all points of $A$, and most from the knob between it and $B$, and converge on $B$. Where they are narrow, the ether is much strained; where wide, the ether is but little strained. Each tube must be looked upon as a tube of unit strain. The nature of the strain of the ether is not known; it is, most probably, some increased motion in a perfect liquid. We must not be surprised at the nature of the strain being unknown. We do not know the nature of the change in a piece of india-rubber when it is strained, nor indeed in any solid, and though the ether is much simpler in structure than india-rubber, it can hardly be wondered at that we have not yet discovered its structure, for it is only within the present century that the existence of the ether was demonstrated, while men have known solids and studied their properties and structure for thousands of years. Any way, there is no doubt that the ether is strained in these tubes of force when A and B are oppositely charged, and that the energy per cubic centimetre of unstrained ether is less than that of strained ether, and that the work done in what is called charging $A$ and $B$ is really done in straining the ether all round them. When the air gap breaks down, and an electric spark takes its place, there is quite a new series of phenomena produced. Suddenly, the strained ether relieves itself, and, in doing so, sets up new motions in itself. The strained state was probably a peculiar state of motion, and in changing back to ordinary ether a new and quite distinct state of motion is set up. This new state of motion all round the conductors is most intense near the spark, and is usually described as an electric current in the conductors and across the spark, or as a rushing of the electric charge from one conductor to the other. The electric current is accompanied by magnetic force in circles round it, and the tubes of magnetic force define the nature of the new movement in the ether as far as we know it. Hitherto, for the sake of simplicity, the existence of this magnetic force has been unnoticed. It is due to a peculiar motion in the ether all round what are called electric currents. The current in fact consists of little else than a line, all round which this movement is going on; like the movement surrounding an electrified body, but also unlike it. Whenever electric forces are changing, or electrified bodies moving, or electric currents running, there this other peculiar motion exists. We have every reason for thinking that this, which may be called the magnetic strain in the ether, as the movement all round electrified bodies was called the electric strain-that this magnetic strain only exists in these three cases: (I) when the electric strain is changing; (2) when electrified bodies are moving; and (3) when electric currents are running. These three may be all cases of one action : certainly the magnetic strain that accompanies each is the same, and it seems most likely that the electric change is only another aspect of the magnetic strain. There are analogies to this in the motion of matter that partly help and partly annoy, because they partly agree and partly will not agree with the etherial phenomena. Take the case described in a former article of a chain transmitting waves. Attention was drawn to the displacement of a link and to its rotation. Now for the analogy: to seem at all satisfactory the first thing that would strike one would be to pay attention to two motions, to the velocity of displacement of the link and to its rotation. This would lead to interminable difficulties in carrying out the analogy. We cannot liken electric strain to a velocity in this direct and simple way, because, what are we to do with a change in the strain which produces the same effects as a continuous current? A change in the strain is all very well, it would be like a change in the velocity, but what about a continuous change in the velocity: we can hardly suppose a velocity continually increasing for ever : we are evidently landed in immediate difficulties. It is better therefore to be content to liken the electric strain to a displacement of the chain link. It seems most likely that it really is a peculiar motion in the ether, but we must be content for the present with the analogy. If we want to drive it further, we must suppose stress in the chain that draws the link back to be due to a motion in the chain or of things fastened to it, and then the changed motions produced by a displacement of the chain might be analogous to the peculiar motions accompanying electric strain. It would lead us too far to work out this analogy. Returning to the simpler case of the displacement of the link representing electric strain, and the velocity of its rotation representing magnetic strain, see how the actions near a Hertzian generator may be likened to what takes place when a wave is being sent along a chain. While the conductors are being slowly charged we must suppose electric strain to be produced in all the surrounding space. This is a comparatively slow action, and as the rate of propagation is very rapid, the electric strain will rise practically simultaneously in the whole neighbourhood, and that it does so is a most important fact to be taken account of in all our deductions from these experiments. This slow charging must be represented by a slow raising of one end of the chain, which raises the rest of it to a great distance apparently simultaneously if the raising be done slowly. Suddenly the air gap breaks. This might

NO. I I 24, VOL. 44] 
be represented by lifting the chain with a weak thread, and by having the end of the chain fastened to a pretty strong spring. When the thread broke the spring would pull the chain back quickly, would pass its position of equilibrium, and thus commence a series of rapid vibrations on each side of this position; the vibrations would gradually die away owing to the energy of the spring being gradually spent, partly on friction in itself, and partly in sending waves along the chain. In actually performing the experiment, an india-rubber tube or limp thin rope is better than a chain when hung horizontally, as the chain is so heavy when it can be hung vertically, a chain does very well. In the description it simplifies matters to describe a chain because it is easier to talk of a link than of a bit of the rope: a link has an individuality that identifies it, while a bit of the rope is so indefinite that it is not so easy to keep in mind any particular bit. Consider now what these waves are, what sort of motion originates them. When the spring first starts, the near parts of the chain move first. What happens to any link? One end of it moves down before the other. What sort of motion, then, has the link? It must be rotating. Thus it is that change in the displacement is generally accompanied by rotation of the links. Thus it is that change in electric strain is accompanied by magnetic strain. The analogy goes farther than this. Each wave thrown off may be described as a wave of displaced or as a wave of rotating links, and the most displaced are at any time the most rapidly rotating links. Just in the same way, what have hitherto been called waves of electric force may also be looked upon as waves of magnetic force. Because there are two aspects in which the motion of the chain may be viewed does not diminish from the essential unity of character of the wave-motion in its waves; and similarly the fact that these Hertzian waves have an electric and a magnetic aspect does not diminish from the essential unity of character of the wave-motion in them. At the same time the two elements, the displacement of a link and the rotation of a link, are quite distinct things ; either might exist without the other; it is only in wave propagation that they essentially co-exist. In the same way electric strain and magnetic strain are quite different things; though in wave-motion, and indeed whenever energy is transmitted from one place to another by means of the ether, they essentially co-exist.

\section{FIVE YEARS' PULSE CURVES.}

$\mathrm{O}^{\mathrm{v}}$

$\mathrm{VER}$ five years ago it occurred to me that there would be considerable interest in keeping a systematic record for some time of the rate of pulsation, i.e. of the number of beats (per minute) of the pulse. I therefore commenced the practice by taking, every night, an observation of my own pulse ; these observations, originally undertaken solely for my own personal interest, have been continued without intermission up to the present time ; and, on throwing the results into a graphic form, I found so close a symmetry and concord between the curves for these five years, that I thought it might be interesting to readers of NATURE to have these results put before them.

First, then, as to the method adopted in these observavations. I count the pulse beats for one minute ${ }^{1}$ every night ${ }^{2}$ before retiring to bed, and invariably while in a standing posture. From the records thus obtained the average for each month is deduced in the usual way, viz. by adding together all the numbers for the month, and dividing by the number of days on which observations were taken. With regard to this important consideration

'Thus avoiding the considerable error that is introiluced by counting for, say, fifteen seconds only, and multiplying.

NO. I I 24 , VOL. 44] -i.e. of the number of observations, since an insufficiency thereof would, of course, greatly vitiate the value of my curves-I may state that during the first four years I omitted to take an observation on only seventeen nights altogether. During the fifth year, I find twenty-one observations missed. Nevertheless, the net data from which the curves are deduced are not quite so abundant as this statement would imply; for, in calculating the monthly average, I have invariably struck out altogether all readings above 79. My reason for this procedure was simply that I wished to obtain a curve showing the normal pulsations; now, anything much above 75 is abnormally high (especially in my own individual instance, for it will be noticed that my pulse is below the usual average of 70), and I can nearly always assign a distinct cause, such as the feverishness caused by a cold, or excitement, or recent exercise ; it therefore appeared to me fairest to knock out altogether the results of such disturbing causes, and since for this purpose an arbitrary line must be drawn somewhere, I decided to draw it at 79. On the other hand, however, I have retained all the other readings, no matter how low they might be, although the "fifties" are very common, and occasionally even the "forties" have been touched. It might, perhaps, be thought that these very low readings should be neglected equally with the very high, but such a course appeared to me altogether illegimate, both because such low readings seemed, judging from their occurrence, to be, so to speak, normally caused, and unassignable to any distinct extraordinary cause known to myself, and also because I should hardly have known where to draw a minimum line. However, I now regret that the readings below 50 , at any rate, were not rejected; but such readings are so extremely rare that they cannot have much influenced the curves. In order that the reader may judge for himself on what data these curves are founded, I have appended a table showing the net number of readings from which each monthly average was drawn, and have also stated (in brackets) the number of readings below 60 included in each month.

Turning now to the curves themselves, this monthly average is shown by the thin line. It is necessary to explain that these curves were drawn by marking the monthly average by a dot on the extreme right of each space representing a month. I was undecided for some time whether to adopt this plan or to mark this dot in the middle of each monthly space ; but after trying both plans I concluded that now adopted to be the simpler. The actual curves were, of course, obtained by connecting all these dots by straight lines.

On examining this manthly curve, it is at once obvious that there is a strong similarity between the five years; clearly every year the curve falls through the spring, until about midsummer, and then rises wonderfully steadily and regularly in every case (except in 1889 ) through the autumn to November or December. On the whole, two maxima seem to be indicated-namely, one in November, followed by a fall, and then by a rise to another maximum in February or January. But it will be noticed that in the winter $1889-90$ there is the unusual phenomenon of a fall through November, and then the two maxima are replaced by an intermediate maximum reached in December. So that here, in spite of the broad concord and regularity, there was rather too much local irregularity to be altogether satisfactory. In the lower portions of the curves, again, there is even more irregularity. Those of 1887 and I 888 (but emphatically the former) are indeed remarkably free from aberration; but in 1886 there is an extraordinarily abrupt and irregular rise through $\mathrm{July}_{\varsigma}$ followed by a compensating fall through August. In I 890 there is an almost identical irregularity in the same two months; while in 1889 we have a remarkable irregularity in the spring. Now these irregularities puzzled me a good deal ; still, in each case 